\title{
A Novel High Strength Porous Hydroxyapatite/Silk Fibroin Composite: Preparation and Characterization
}

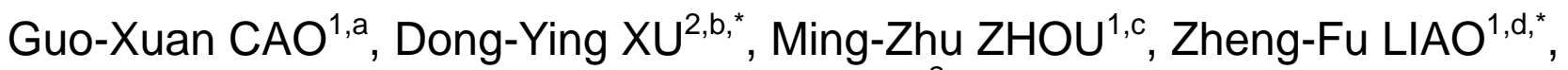 \\ Yu-Dong WANG ${ }^{2, e}$
}

${ }^{1}$ Guangdong University of Technology, School of Materials and Energy, Guangzhou 510006

${ }^{2}$ Guangdong Pharmaceutical University, School of Chemistry and Chemical Engineering, Guangzhou 510006

acaogx11@163.com, ${ }^{\mathrm{b}}$ xudongying123@163.com, ${ }^{\mathrm{c}} \mathrm{mzchou@126.com,}$, ${ }^{\mathrm{d}}$ liaozhengfu@ 126.com, ${ }^{\mathrm{e}}$ dong3 295@sohu.com

${ }^{*}$ Corresponding author

Keywords: Hydroxyapatite (HA), Silk fibroin (SF), Scaffold, High strength.

\begin{abstract}
High strength Bombyx mori silk fibroin (SF) and hydroxyapatite (HA) composite scaffold was obtained by using co-precipitation and salt fractionation method. SEM and mechanical test were used to investigate the structure and properties of the composite scaffolds. The results showed that when the content of $\mathrm{NaCl}$ were $10 \mathrm{wt} \%$, the scaffolds performed the best mechanical properties (compressive strengths and modulus), which reached 12MPa and 598MPa, respectively. The results of SEM suggested that the composites present porous morphologies structure.
\end{abstract}

\section{Introduction}

Human bones are formed by a series of complex events involving mineralization with calcium phosphate in the form of hydroxyapatite on extracellular matrix proteins primarily consisting of collagen type $\mathrm{I}^{[1,2]}$. Recently, Composite material scaffold research has attracted extensive attention. In the present study, biomimetic growth of hydroxyapatite on suitable porous organic matrix was explored to generate organic/inorganic composites as scaffolds for bone tissue engineering. For tissue engineering, the $3 \mathrm{D}$ porous structure of the scaffold is usually necessary, which enables the body fluid and blood to enter into the pores so that the further bony metabolism and in-growth can be promoted in implants. The mechanical strength also plays an important role in the scaffold utility, especially when considered for the bone tissue regeneration ${ }^{[3-5]}$.

The aim of this study was to prepare a SF/HA composite porous scaffold using a salt fractionation method. The micro-structural morphologies, porosity, and mechanical properties of the scaffolds were characterized.

\section{Experiment part}

\section{Materials and Methods}

Bombyx mori silkworms were obtained from Guangxi province, China. $\mathrm{Na}_{2} \mathrm{CO}_{3}, \mathrm{CaCl}_{2}$, $\left(\mathrm{NH}_{4}\right)_{2} \mathrm{HPO}_{4}$, and other reagents are analytical grade. Deionized water was used throughout the experiment. The diameter of $180-250 \mu \mathrm{m}$ of $\mathrm{NaCl}$ was achieved by griddle with meshes size of 60 and 80.

The HA/SF composites were prepared according to the method reported by Yashi $\mathrm{Jin}^{[6]}$ with some modifications.

Scaffold materials preparation: Mixing $\mathrm{NaCl}$ with $\mathrm{HA} / \mathrm{SF}$ composite obtained before by mould, then press into cylinders (diameter $5 \mathrm{~mm}$, about $10 \mathrm{~mm}$ high). The content of $\mathrm{NaCl}$ ranged from 0wt.\% to $20 \mathrm{wt} . \%$. Soak columnar scaffolds in ethanol/water solution for 4 days to remove the $\mathrm{NaCl}$ in scaffolds.

The morphologies of SF/HA composites were characterized by scanning electron microscopy 
(SEM, S-3400N, Hitachi, Japan).

Compressive test was performed at room temperature on the as-prepared samples using an Universal Material Testing Machine (CMT 5504) ( $n=5)$. Elastic modulus, fracture stress, and ultimate strain were calculated from the stress-strain cures. For compression testing, a crosshead speed of $1 \mathrm{~mm} / \mathrm{min}$ was used. Hysteresis (or energy lost due to permanent deformation) was measured by subjecting the sample to a loading and unloading cycle $\mathrm{e}^{[7-9]}$.

\section{Results and Discussion}

In the process of HA crystal forming and growth, the SF chain presents some template effects. The SF macromolecule chains in the composites took the $\beta$-sheet crystal structure, and induced the HA crystal growth. The SF molecule chains enhanced three-dimensional network, which extended throughout the composites, is formed via the crosslink between HA clusters and SF fibrils ${ }^{[10]}$. Fig.1 showed the morphologies structures of SF/HA composites regulated by different content of $\mathrm{NaCl}$ particles. It was found that the size of the pore seems to be at the same order of magnitude. However, with the increase of the content of $\mathrm{NaCl}$, the size of the pore tends to be more uniform and regular, which would provide the high strength of composites.

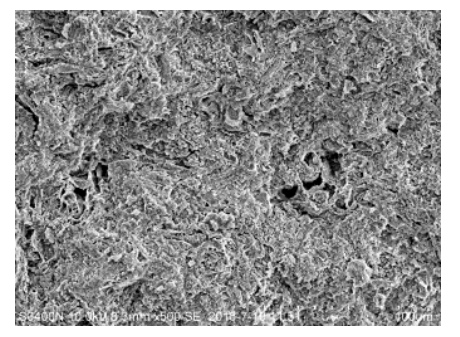

a

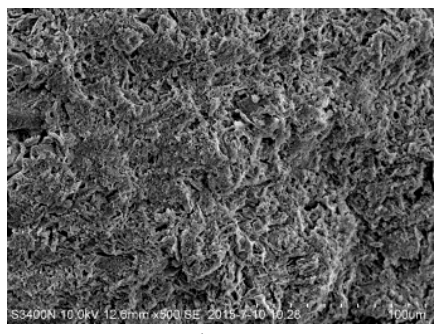

b

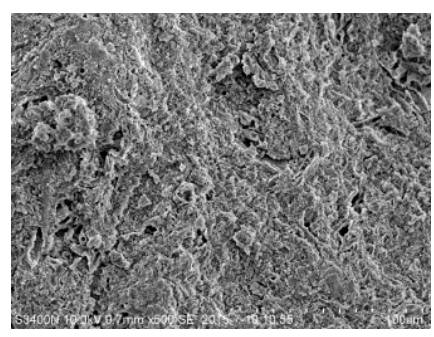

$\mathrm{c}$

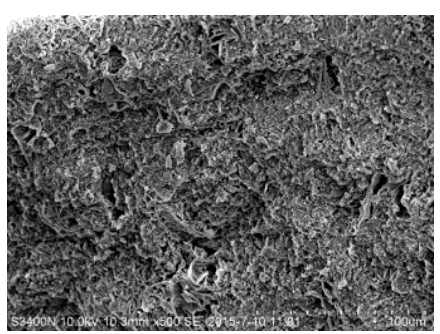

d

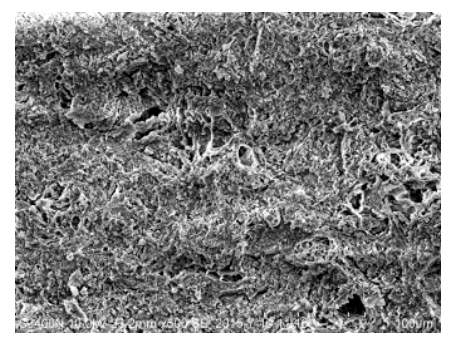

e

Fig.1 Morphologies of HA/SF Composites Based on Different $\mathrm{NaCl}$ Contents: a, b, c, d and e are $0 \%, 5 \%, 10 \%, 15 \%, 20 \%$

Fig.2 showed that the changes of compressive strength and compressive modulus of HA/SF composites based on different $\mathrm{NaCl}$ content. The result shows that the average compressive strengths and modulus increase with the increase of $\mathrm{NaCl}$ content before $10 \mathrm{wt} . \%$. When $\mathrm{NaCl}$ content increased from $10 \mathrm{wt} . \%$ to $20 \mathrm{wt} . \%$, the compressive strengths and modulus of the composites scaffold decreased. Compare with the SEM images, it shows that a certain extent of pore density can increase the mechanical properties of scaffold. However, these values were over three orders of magnitude greater than those measured for analogous collagen-based scaffolds $(0.015 \text { and } 0.15 \mathrm{MPa} \text {, respectively })^{[11]}$. And four kinds of samples were reach the mechanical tolerances observed in cancellous bone ${ }^{[12,13]}$. However, undried SF/HA composites scaffold displayed both brittle and ductile deformation during compression. 

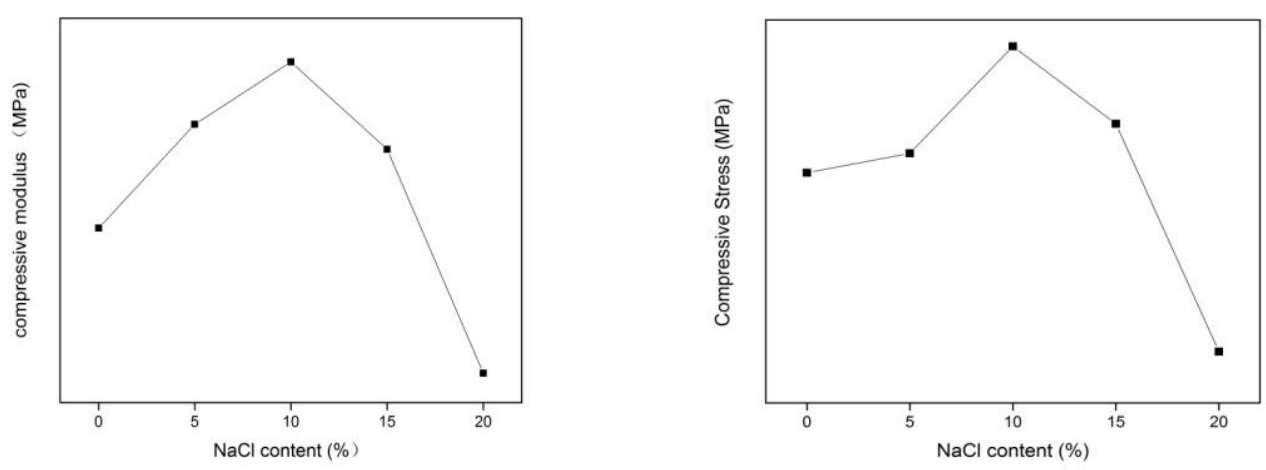

Fig.2 Compressive Strength and Compressive Modulus of SF/HA Composites Based on Different $\mathrm{NaCl}$ Contents

In contrast, a commercially available sintered calcium phosphate scaffold had a much lower average compressive strength of $4 \pm 1 \mathrm{MPa}^{[14]}$, and fractured during compressive overload. It suggests that the SF/HA composite should have significant advantages over currently available ceramic scaffolds in surgical procedures where high mechanical tolerance is required.

\section{Conclusion}

The SF/HA composite was fabricated by means of co-precipitation and salt fractionation. When $\mathrm{NaCl}$ content were $10 \mathrm{wt} . \%$, the composites performed best compressive strength and modulus. The work primarily investigated the structure and properties of SF/HA composites. The high strength $\mathrm{SF} / \mathrm{HA}$ composite may potential materials in the field of scaffolds materials for bone tissue repair, and the relative works is ongoing in our laboratory.

\section{Acknowledgements}

This work was supported by the Natural Science Foundation of Guangdong Province (Grant No.2014A030313513). The authors also thank the Joint Natural Sciences Fund of the Department of Science and Technology and the First Affiliated Hospital of Guangdong Pharmaceutical University (Grant No. GYFYLH201323).

\section{References}

[1] Hyeon Joo Kim, Ung-Jin Kim, Hyun Suk Kim, Chunmei Li, Masahisa Wada, Gary G. Leisk, David L. Kaplan. Bone tissue engineering with premineralized silk scaffolds. Bone 2008; 42:1226-1234.

[2] Mehdi Sadat-Shojai, Mohammad-Taghi Khorasani, Ehsan Dinpanah-Khoshdargi, Ahmad Jamshidi. Synthesis methods for nanosized hydroxyapatite with diverse structures. Acta Biomaterialia 2013; 9:7591-7621.

[3] Lin Liu, Jinying Liu, Mingqi Wang, Sijia Min, Yurong Cai, Liangjun Zhu,Juming Yao. Preparation and characterization of nano-hydroxyapatite/silk fibroin porous scaffolds, Journal of Biomaterials Science, Polymer Edition 2008; 19:3, 325-338, DOI: 10.1163/156856208783721010.

[4] Hua Liu, Guo Wei Xu, Ya Fei Wang, Hong Shi Zhao, Si Xiong, et al.Composite scaffolds of nano-hydroxyapatite and silk fibroin enhance mesenchymal stem cell-based bone regeneration via the interleukin 1 alpha autocrine/paracrine-signaling loop. Biomaterials 2015; 49:103-112.

[5] Joseph R.Woodard, Amanda J.Hilldore, Sheeny K. Lan, C.J.Park, Abby W.Morgan, et al. The 
mechanical properties and osteoconductivity of hydroxyapatite bone scaffolds with multi-scale porosity. Biomaterials 2007; 28: 45-54.

[6] Yashi Jin, Banani Kundu, Yurong Cai, Subhas C. Kundu, Juming Yao. Bio-inspired mineralization of hydroxyapatite in 3D silk fibroin hydrogel for bone tissue engineering. Colloids and Surface B: Biointerfaces 2015; 134:339-345.

[7] John A Kilion, Luke M Geever, Declan M Devine and Clement L Higginbotham. Fabrication and in vitro biological evaluation of photopoymerisable hydroxyapatite hydrogel composites for bone regeneration. Journal of Biomaterials Applications 2014; vol. 28(8): 1275-1283.

[8] S. Baradaran, E. Moghaddm, W.J. Basirun, M. MEHRALI, et al. Mechanical properties and biomedical applcations of a nanotube hydroxyapatite-reduced grapheme oxide composite. Carbon 2014; 69: 32-45.

[9] Chunyu Chang, Na Peng, Meng He, Yoshikuni Teramoto, Yoshiyuki Nishio, Lina Zhang. Fabrication and properties of chitin/hydroxyapatite hybrid hydrogels as scaffold nano-materials. Carbo hydrate Polymers 2013; 91: 7-13.

[10] Li Wang, Rei Nemoto, Mamoru Senna. Changes in microstructure and physic-chemical properties of hydroxyapatite-silk fibroin nanocomposite with varying silk fibroin content. Journal of the European Ceramic Society 2004; 24:2707-2715.

[11] R. Murugan, S. Ramakrishna. Development of nanocomposites for bone grafting. Composites Science and Technology 2005; 65:2385-2406.

[12] Andrew M. Collins, Nick J.V.Skaer, Tom Gheysens, David Knight, Caroline Bertram, Helmtrud I. Roach, Richard O.C. Oreffo, Sonja Von-Aulock, Teodora Baris, John Skinner, Stephen Mann. Bone-like Resorbable Silk-based Scaffolds for Load-bearing Osteoregenerative Applications. Adv. Mater 2009; 21:75-78.

[13] Hyeon Joo Kim, Ung-Jin Kim, Hyun Suk Kim, Chunmei Li, Masahisa Wada, Gary G. Leisk, David L. Kaplan. Bone tissue engineering with premineralized silk scaffolds. Bone 2008; 42:1226-1234.

[14] Andrew M. Collins, Nick J.V.Skaer, Tom Gheysens, David Knight, Caroline Bertram, Helmtrud I. Roach, Richard O.C. Oreffo, Sonja Von-Aulock, Teodora Baris, John Skinner, Stephen Mann. Bone-like Resorbable Silk-based Scaffolds for Load-bearing Osteoregenerative Applications. Adv. Mater 2009; 21:75-78. 